\title{
A Qualidade de Vida de Pessoas com Deficiência Mental Leve
}

\author{
Fernanda Saviani-Zeoti ${ }^{1}$ \\ Eucia Beatriz Lopes Petean \\ Universidade de São Paulo
}

\begin{abstract}
RESUMO - Não é prática comum dar voz a pessoas com deficiência, mesmo quando se trata da investigação de sua própria qualidade de vida. Assim, este estudo teve por objetivo conhecer a opinião de 15 adultos com deficiência mental leve em relação a sua qualidade de vida e a opinião de seus cuidadores também a esse respeito, por meio de um instrumento que avalia a qualidade de vida (WHOQOL-Bref). Os dados foram analisados estatisticamente e comparados. Os resultados mostram que a diferença entre as avaliações foi pequena nas questões referentes à satisfação com os domínios físico, psicológico, das relações sociais e do meio ambiente. A avaliação feita pelas pessoas com deficiência foi apenas ligeiramente superior àquela feita por seus cuidadores. Não houve diferença estatisticamente significativa entre as avaliações, do que se conclui que as pessoas com deficiência mental são capazes de falar de suas próprias vidas de maneira positiva e bastante realista.
\end{abstract}

Palavras-chave: qualidade de vida; deficiência mental; cuidador.

\section{Quality of Life of People with Mild Intellectual Disability}

\begin{abstract}
People with intellectual disability are often neglected even though it is a question of their own quality of life. Thus, this study aimed to know the opinion of 15 adults with mild intellectual disability related to their quality of life and the opinion of their caregivers about it with an instrument that evaluates quality of life (WHOQOL-Bref). The data were statistically analyzed and compared. The results show that the difference between the evaluations was small in the questions concerning satisfaction with the physical, psychological, social relation and environment welfares. People with disability' evaluation was few higher than their caregiver's evaluation. There weren't significant statistics difference between evaluations, so that we conclude that the people with intellectual disability are able to talk about their own lives with an optimistic view and a very realistic way.
\end{abstract}

Key words: quality of life; intellectual disability; caregiver.

O termo qualidade de vida foi empregado pela primeira vez em 1964, quando o presidente dos Estados Unidos, Lyndon Johnson, declarou que "os objetivos não podem ser medidos através do balanço dos bancos. Eles só podem ser medidos através da qualidade de vida que proporcionam às pessoas" (Fleck, Leal \& cols., 1999, p. 20). Desde então, o seu significado vem sendo estudado e estruturado por diversas áreas do conhecimento.

A Organização Mundial de Saúde (OMS), em 1995, definiu o conceito de qualidade de vida, fundamentando-o em três pressupostos: subjetividade, multidimensionalidade e presença de dimensões positivas e negativas. Assim, definiu-se qualidade de vida como "a percepção do indivíduo de sua posição na vida, no contexto da cultura e sistema de valores nos quais ele vive e em relação aos seus objetivos, expectativas, padrões e preocupações" (Whoqol Group citado por The Whoqol Group, 1998b, p. 1570).

Nos últimos anos, as pesquisas que têm como tema a qualidade de vida abrangem diversas populações: usuários de serviços de saúde (Schalock, 2000; Schalock, Bonham \& Marchand, 2000; Velanovich, 2001); pessoas que possuem doenças graves (Bullinger \& cols., 1996), doenças genéticas ou hereditárias (Bullinger \& cols., 2002; Kuhnle, Bullinger \& Schwarz, 1996; Mancuso, Peterson \& Charlson, 2001) ou

1 Endereço: Rua Santos Dumont, 1185, Apt. 21, Sumarezinho, SP, Brasil 14050-060.E-mail: fersaviani@bol.com.br doenças crônicas e letais (Almeida \& Loureiro, 2000; Sabaz, Cairns, Lawson, Bleasel \& Bye, 2001; Vickery \& cols., 2001; Stockler, Osoba, Corey, Goodwin \& Tannock, 1999); e, mais recentemente, as pesquisas também investigam a qualidade de vida de pessoas idosas (Berglund \& Ericsson, 2003; Walsh, 2002).

No entanto, foi somente a partir da década de 1990 que o tema vem sendo o foco de pesquisas e aplicações nas áreas da Educação/Educação Especial, cuidados com a saúde (física e comportamental), serviços sociais (deficiências e faixa etária) e famílias (Schalock, 2004).

Em sua maioria, as pesquisas que investigam a qualidade de vida de deficientes mentais apresentam a opinião dos cuidadores e/ou familiares sobre a pessoa com deficiência ou sobre si mesmos (Cummins, 2002; Hatton \& Ager, 2002; Sabaz \& cols., 2001; Walden, Pistrang \& Joyce, 2000; Walsh, Heller, Schurf \& Valk, 2001), encontrando-se um número reduzido de estudos referentes à opinião dos próprios deficientes a respeito de suas vidas.

As pesquisas que têm a própria pessoa com deficiência como participante, em geral investigam a percepção que ela possui de sua própria vida, relacionamentos e ambiente social, por entrevistas elaboradas pelos próprios pesquisadores ou instrumentos genéricos, não existindo estudos que utilizem instrumentos específicos que tratem da qualidade de vida de pessoas com deficiência mental.

Com isso, pode-se afirmar que, de maneira geral, conhecer a opinião de deficientes, dar voz a eles, não é prática 
comum socialmente e principalmente em pesquisas. Este fato é agravado quando se trata do deficiente mental, pois sabe-se que dificuldade de compreensão e incapacidade para perceber sua situação são algumas das preconcepções que atravessam o tempo e impedem a realização de estudos que o tenham como sujeito da ação.

Entretanto, sabe-se que pessoas com deficiência mental são capazes de sentir, pensar sobre seus sentimentos e expressá-los: quando crianças, por desenhos e brincadeiras, e, depois de adultos, pela fala (Assumpção Jr., 1991).

Dessa forma, o objetivo deste estudo foi conhecer a percepção de pessoas com deficiência mental leve, suas expectativas e preocupações em relação a sua própria qualidade de vida e comparar essa percepção com aquela que os cuidadores têm a respeito da qualidade de vida das pessoas cuidadas.

\section{Método}

Para a realização desta pesquisa, houve a participação de 30 pessoas, sendo 15 com deficiência mental leve, com idade igual ou superior a 20 anos, de ambos os sexos, independentemente do nível socioeconômico, e outras 15 seus respectivos cuidadores mais próximos. Para se garantir o anonimato dos participantes, foram atribuídos números a cada uma das duplas, sendo a letra D referente à pessoa com deficiência e a letra $\mathrm{C}$ ao seu cuidador.

A seleção foi feita em um Centro de Educação Especial da cidade de Ribeirão Preto e por indicações de profissionais da área conhecedores dos objetivos da pesquisa. O contato inicial com os participantes foi realizado por telefone e/ou pessoalmente.

Nesta pesquisa, adotaram-se as definições da OMS para deficiência mental e deficiência mental leve. Deficiência mental é a "limitação das funções mentais gerais, necessárias à compreensão e interpretação integradas a diferentes funções mentais, incluindo todas as funções cognitivas e seu desenvolvimento ao longo da vida" (World Health Organization, 2001, p. 51). As pessoas que apresentam deficiência mental leve, de acordo com a OMS, são caracterizadas como indivíduos que podem adquirir aptidões práticas e de leitura e aritmética, com uma educação especial, e que podem ser orientados para uma integração especial (Organização Mundial de Saúde, 1995).

Para a avaliação da qualidade de vida nesta pesquisa, foi utilizado o World Health Organization Quality of Life, em sua forma abreviada (WHOQOL-Bref). Esse instrumento foi desenvolvido pela Organização Mundial de Saúde e possui versão validada e adaptada para nosso idioma e cultura por Fleck e cols. (2000), contendo 26 questões divididas em quatro domínios: físico, psicológico, relações sociais e meio ambiente. De acordo com Fleck, Louzada e cols. (1999), o instrumento é auto-explicativo e pode ser auto-administrado, assistido pelo entrevistador ou, ainda, administrado pelo entrevistador.

Para o grupo dos cuidadores, esse instrumento foi autoadministrado e utilizado para que respondessem sobre a qualidade de vida da pessoa com deficiência por quem são responsáveis; e, para tanto, as questões foram adaptadas da primeira para a terceira pessoa. Para o grupo das pessoas com deficiência, constatou-se, mediante o pré-teste, a necessidade da aplicação do instrumento com a ajuda da pesquisadora, que lia as questões, assim como opções de resposta, e os participantes as respondiam. Algumas adequações de linguagem foram necessárias para que o material fosse compreendido o mais plenamente possível. Isso não foi feito de maneira fixa, mas variável, de acordo com cada um dos participantes.

As coletas com cada uma das duplas (pessoas com deficiência e cuidadores) foram realizadas no mesmo dia e local, mantendo a maior privacidade possível para cada grupo, ou seja, em nenhum momento os participantes permaneceram na presença um do outro enquanto eram entrevistados. Todos os participantes assinaram o Termo de Consentimento. $\mathrm{O}$ termo referente à pessoa com deficiência foi assinado por ela e por seu respectivo cuidador, seu responsável legal.

Os dados obtidos no WHOQOL-Bref foram pontuados utilizando-se o programa estatístico SPSS, de acordo com a Organização Mundial de Saúde (The Whoqol Group, 1998a).

Realizaram-se estudos descritivos visando estabelecer parâmetros para a comparação dos dados obtidos em cada uma das avaliações: das pessoas com deficiência mental e dos cuidadores, avaliando a qualidade de vida das pessoas com deficiência por quem são responsáveis.

Essa comparação foi feita mediante verificação de diferenças entre os grupos, em relação às avaliações nos quatro domínios do WHOQOL-Bref. Para tanto, foi aplicada a prova estatística não-paramétrica U de Mann-Whitney, com um nível de significância igual ou menor que 0,05 .

Além dessa comparação, foram conjugados entre si os resultados apresentados pelo WHOQOL-Bref e os fatores que caracterizam os participantes desta pesquisa (renda familiar, idade e tempo de cuidados), por meio da Técnica de Quadrantes descrita por Figueiredo (1994) e utilizada por Souza (2001).

\section{Resultados}

Entre as pessoas com deficiência participantes desta pesquisa, oito são do sexo masculino e sete do sexo feminino. A grande maioria ( $87 \%$ ) tem entre 20 e 30 anos e somente $13 \%$ possuem mais de 45 anos de idade. Das pessoas estudadas, $40 \%$ têm Síndrome de Down, uma (7\%) tem paralisia cerebral e as demais apresentam algum tipo de atraso cognitivo, mas apenas $60 \%$ de todas elas sabem qual é a deficiência que possuem. Quanto à escolaridade, $80 \%$ das pessoas com deficiência participantes estudam atualmente, cursando supletivo ou aulas particulares e os outros $20 \%$ não estudam mais. Todos os participantes sabem escrever o próprio nome e lêem frases simples, porém, possuem dificuldade para ler e compreender textos mais elaborados. E, ainda, 54\% deles trabalham, 33\% já trabalharam algum dia e apenas $13 \%$ nunca trabalharam.

Em relação aos cuidadores, a maioria (73\%) são as mães, sendo que, na população estudada, há um pai, uma irmã, uma mãe adotiva e uma tia materna, que são os cuidadores das pessoas com deficiência e seus responsáveis legais. Dos cuidadores participantes, $47 \%$ têm 50 anos ou menos, enquanto os outros $53 \%$ têm mais que 50 anos de idade. A maioria é casada (66\%), 20\% são viúvas, uma (7\%) é solteira 
e uma $(7 \%)$ é separada. Somente dois deles possuem nível superior de escolaridade, sete fizeram o ensino médio e os demais o ensino fundamental completo ou incompleto. A renda familiar é muito variada, indo de um até 17 salários mínimos, sendo que $40 \%$ têm uma renda inferior ou igual a quatro salários e os outros $60 \%$, uma renda igual ou superior a cinco salários mínimos. Por serem seus familiares, o tempo que os cuidadores exercem este papel coincide com a idade da pessoa com deficiência, diferindo em apenas um dos casos,

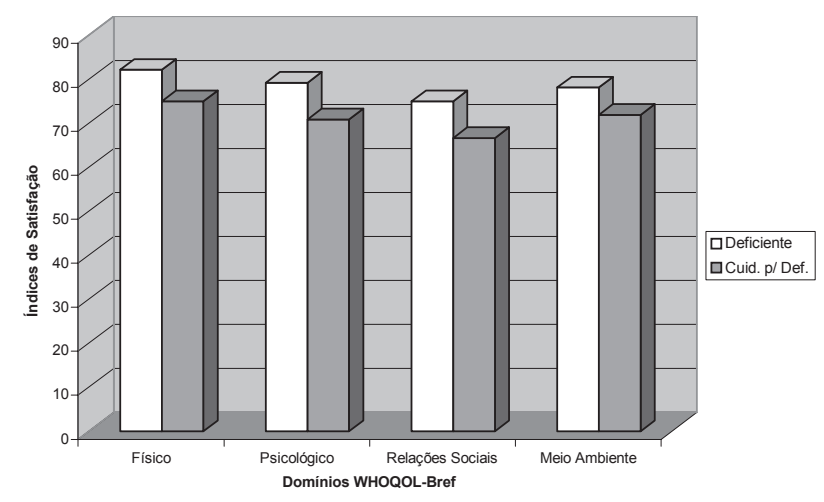

Figura 1. Medianas da distribuição dos índices de satisfação nos domínios de avaliação da qualidade de vida (WHOQOL-Bref), feita pelas pessoas com deficiência e seus respectivos cuidadores.

em que a irmã exerce a função de cuidadora do irmão desde a morte da mãe, há 12 anos.

\section{Avaliação da qualidade de vida (WHOQOL-Bref)}

O tratamento dos dados obtidos na aplicação do WHOQOL-Bref resultou nos índices de satisfação de cada um dos grupos, nos quatro domínios (Figura 1).

A Figura 1 apresenta os dados a partir das medianas da distribuição dos índices de satisfação nos domínios do WHOQOL-Bref. Pode-se observar uma maior satisfação em todos os domínios por parte das pessoas com deficiência, em comparação à avaliação que os cuidadores fazem da vida dessas pessoas. Ou seja, os índices de satisfação que os cuidadores apresentaram em relação à qualidade de vida das pessoas com deficiência estão sempre abaixo da autoavaliação dessas pessoas.

As pessoas com deficiência tiveram uma maior satisfação com os aspectos que integram o domínio físico e a menor satisfação apresentada se refere aos fatores avaliados pelo domínio relações sociais. Quando os cuidadores avaliam a qualidade de vida das pessoas com deficiência, os domínios com maior e menor satisfação são os mesmos.

Para verificar a diferença entre os grupos deste estudo, aplicou-se a prova U de Mann-Whitney:
Na Tabela 1, observa-se que as pessoas com deficiência mental desta pesquisa apresentaram avaliações mais positivas, em todos os domínios do WHOQOL-Bref (valores de $X p$ ), do que aquelas feitas por seus cuidadores a respeito da vida delas. Porém, essas diferenças entre as avaliações dos dois grupos não são significativas ( $p>0,05)$.

Além dessa comparação, por meio da Técnica de Quadrantes (Figueiredo, 1994), realizou-se a conjugação dos dados obtidos com as avaliações de qualidade de vida e das seguintes variáveis: idade, renda familiar e tempo, em anos, durante o qual os cuidadores exercem a função de cuidar.

\section{Relações entre qualidade de vida das pessoas com deficiência mental e fatores que caracterizam esses participantes (idade e renda familiar)}

A definição dos quadrantes foi feita mediante posicionamento das medianas das distribuições dos índices de satisfação das pessoas com deficiência, nos domínios que constituem o WHOQOL-Bref, nas ordenadas, e das variáveis nas abscissas:

\section{Domínios do WHOQOL-Bref e Idade da Pessoa com Deficiência Mental}
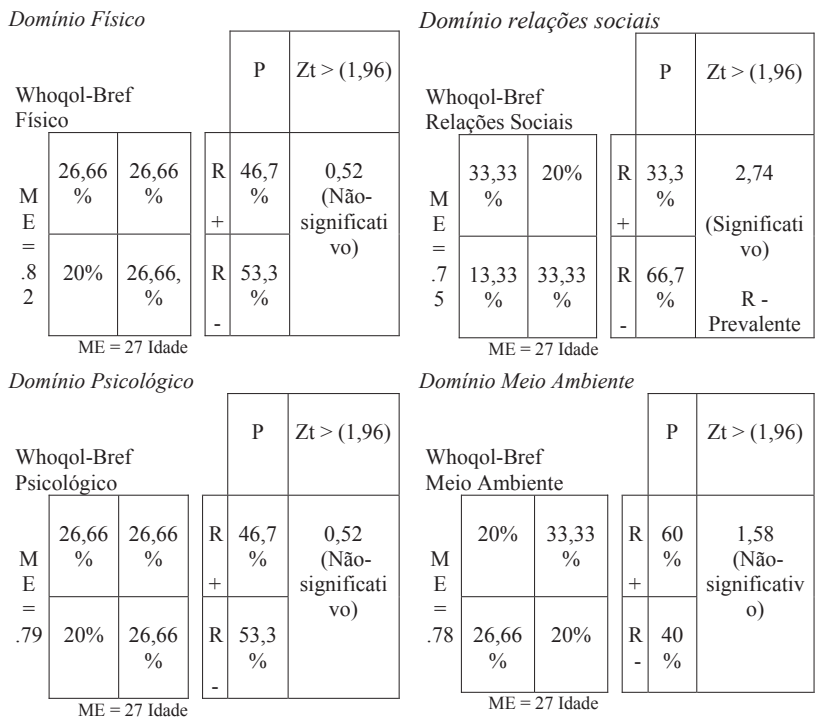

Os dados resultantes mostram que há uma relação significativa entre a idade das pessoas com deficiência participantes e os seus índices de satisfação no domínio físico do WHOQOL-Bref.

Dessa forma, aquelas pessoas que são mais velhas apresentam-se mais satisfeitas com os fatores do seu meio ambiente relacionados com a sua qualidade de vida.

Tabela 1. Resultados do teste U de Mann-Whitney aplicado aos índices de satisfação nos domínios do WHOQOL-Bref obtidos pelos dois grupos: deficiente e cuidador.

\begin{tabular}{|c|c|c|c|c|c|c|c|c|}
\hline & \multicolumn{2}{|c|}{ Físico } & \multicolumn{2}{|c|}{ Psicológico } & \multicolumn{2}{|c|}{ Relações Sociais } & \multicolumn{2}{|c|}{ Meio Ambiente } \\
\hline & Def. & Cuid. & Def. & Cuid. & Def. & Cuid. & Def. & Cuid. \\
\hline$X p$ & 16,80 & 14,20 & 18,63 & 12,37 & 17,83 & 13,17 & 17,90 & 13,10 \\
\hline$N$ & 15 & 15 & 15 & 15 & 15 & 15 & 15 & 15 \\
\hline$p$ & \multicolumn{2}{|c|}{0,4} & \multicolumn{2}{|c|}{0,6} & \multicolumn{2}{|c|}{0,2} & \multicolumn{2}{|c|}{0,1} \\
\hline
\end{tabular}


Domínios do WHOQOL-Bref e Renda Familiar
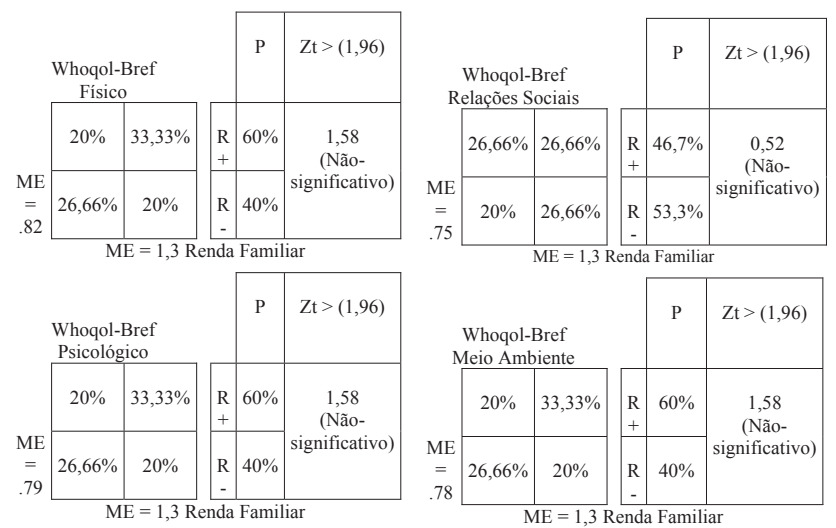

A partir desses resultados, observa-se que não houve relações significativas entre os índices de satisfação das pessoas com deficiência mental com a própria qualidade de vida e o fator renda familiar que caracteriza essa população.

\section{Discussão}

Para a seleção das 15 duplas (pessoa com deficiência mental leve e seu cuidador) que participaram desta pesquisa, optou-se por entrar em contato com um Centro de Educação Especial, por ser esse o lugar em que se encontra o maior número de pessoas com deficiência mental na cidade de Ribeirão Preto. A busca por esse tipo de população em ambientes escolares ou centros especializados é a forma mais utilizada pelos pesquisadores que abordam o tema da deficiência mental (Emerson, Robertson \& Wood, 2004; Gregory, Robertson, Kessissoglou, Emerson \& Hatton, 2001; Kraemer, McIntyre \& Blancher, 2003; Schwartzman, Martin, Yu \& Whiteley, 2004; Schwartz \& Rabinovitz, 2003).

Foram encontrados, na literatura, somente dois estudos em que os próprios adultos deficientes mentais relataram sua satisfação com alguns aspectos de suas vidas (Gregory \& cols., 2001; Schwartz \& Rabinovitz, 2003). Nessas pesquisas, a população teve número equilibrado de participantes quanto ao sexo. $\mathrm{O}$ mesmo ocorreu neste trabalho, em que participaram oito homens e sete mulheres com deficiência mental, embora o sexo não tenha sido critério de participação quando o projeto foi elaborado.

Ao se tratar da questão do trabalho, percebe-se que alguns cuidadores só consideram como trabalho aquilo que é formal ou resulta em um salário, enquanto as próprias pessoas com deficiência mental mostram-se satisfeitas com o que fazem, considerando realmente como trabalho as oficinas pedagógicas que freqüentam no Centro de Educação, pois recebem algum dinheiro pelas peças que vendem. Talvez isso ocorra porque uma das grandes barreiras, quanto à expectativa por parte dos pais, está no status que o trabalho ou ocupação dará ao filho (Regen, Ardore \& Hoffmann, 1993).

Schwartz e Rabinovitz (2003) tiveram a população de seu estudo dividida em dois grupos: pessoas com deficiência mental leve (48\%) e moderada (52\%). Sessenta e dois por cento de todos eles trabalhavam em contextos protegidos (instituição) e os demais no comércio da cidade. Na presente pesquisa, $50 \%$ da população trabalha atualmente. Destes, um trabalha no comércio, os demais encontram-se em oficinas profissionalizantes ou pedagógicas de um Centro de Educação Especial da cidade. Esse dado demonstra a dificuldade de inserção do deficiente no mercado de trabalho no Brasil.

Entre as pessoas que participaram desta pesquisa, a maioria não tem um diagnóstico definido para sua deficiência mental, sendo que seis apresentam Síndrome de Down e um paralisia cerebral atáxica. Em pesquisas que trabalham com familiares/cuidadores de pessoas com deficiência mental, também existe uma grande parcela da população que não tem diagnóstico definido e os que o possuem, na maioria das vezes, apresentam Síndrome de Down, Paralisia Cerebral e/ou Autismo (Kraemer \& cols., 2003; Pruchno \& Meeks, 2004; Shu, Lung \& Huang, 2002; Walden \& cols., 2000). Talvez isso ocorra pela facilidade de diagnóstico e incidência desses quadros clínicos na população e porque, dificilmente, uma pessoa que os apresente fique sem definição diagnóstica. De maneira geral, apesar da falta de diagnóstico, as pessoas com deficiência que participaram deste estudo sabem quais as dificuldades que possuem, demonstrando assim a consciência que têm de suas limitações e potencialidades.

Com relação ao grau de escolaridade, $13 \%$ dos participantes com deficiência não estudam mais e $7 \%$ fazem aulas particulares. Todos os outros frequientam o ensino de jovens e adultos (supletivo) em escola especializada. No estudo de Kraemer e cols. (2003), em que familiares foram entrevistados a respeito da qualidade de vida dos jovens com deficiência mental sob seus cuidados, foi relatado que $45 \%$ deles tinham o ensino fundamental completo, porém $55 \%$ deles freqüentavam classes especiais. Esses dados encontrados pelos pesquisadores e também pelo presente estudo demonstram que, independentemente da idade, a permanência em instituições educacionais formais é importante, não só para os familiares como também para as pessoas com deficiência.

Em relação aos cuidadores que participaram desta pesquisa, a grande maioria são as próprias mães (73\%), fato que muito freqüentemente ocorre em pesquisas na área (Kraemer \& cols., 2003; Schwartz \& Rabinovitz, 2003; Shu $\&$ cols., 2002). Provavelmente isso ocorra devido ao papel que a figura materna possui, o de ser a referência de cuidados dispensados aos filhos, principalmente àqueles mais frágeis e dependentes (Santi, 2003).

Ainda nos estudos realizados com cuidadores de pessoas com deficiência mental, a maioria da população participante é casada (Pruchno \& Meeks, 2004; Walden \& cols., 2000) e apresenta uma idade média próxima a 50 anos (Kraemer \& cols., 2003; Walden \& cols., 2000), características que a população desta pesquisa também apresentou.

Ainda entre os participantes deste estudo, apenas dois cuidadores possuem o ensino superior, todos os outros cursaram até o ensino médio ou somente o fundamental. Nas pesquisas realizadas com pessoas com deficiência mental, a escolaridade dos cuidadores não é algo muito explorado, no entanto, as que apresentam esse dado mostram, geralmente, que a maioria da população tem como maior grau de instrução o ensino médio e muito poucos possuem o ensino superior (Hastings, Allen, McDermott \& Still, 2002; Pruchno \& Meeks, 2004).

Como já dito anteriormente, neste estudo foi utilizado o questionário que avalia a qualidade de vida geral, em sua forma abreviada (WHOQOL-Bref). Esse questionário da 
Organização Mundial de Saúde tem sido amplamente utilizado em pesquisas internacionais que abordam o tema da qualidade de vida. Atualmente, esse instrumento (WHOQOL-Bref) tem sido utilizado no Brasil para investigar a qualidade de vida das mais variadas populações: pacientes psiquiátricos (Carvalho, Lima, Gonçalves, Barbosa \& Fagundes, 2002; Fleck \& cols., 2002); com enfermidades crônicas e/ou terminais (Michelone \& Santos, 2004); que passaram por cirurgias (Bittencourt, Alves Filho, Mazzali \& Santos, 2004; Moreno \& Lopes, 2002); e até estudantes universitários (Saupe, Nietche, Cestari, Giorgi \& Krahl, 2004).

Nesta pesquisa, as pessoas com deficiência mental leve foram as que apresentaram os maiores índices de satisfação com a própria qualidade de vida. Apresentaram uma avaliação significativamente superior nos domínios psicológico (sentimentos positivos, pensar, aprender, memória e concentração, auto-estima, imagem corporal e aparência, sentimentos negativos, e espiritualidade, religião e crenças pessoais) e meio ambiente (segurança física e proteção, ambiente no lar, recursos financeiros, cuidados de saúde e sociais, oportunidade de adquirir novas informações e habilidades, oportunidades de recreação/lazer, ambiente físico e transporte).

Os cuidadores, por sua vez, apresentam-se menos satisfeitos em relação à qualidade de vida das pessoas sob seus cuidados. Essas diferenças entre as avaliações da qualidade de vida das pessoas com deficiência mental não são estatisticamente significativas, sendo que os dois grupos demonstraram maior e menor satisfação com os aspectos psicológicos e de relacionamento social, respectivamente.

No caso das pessoas com deficiência, o único fator que se apresenta significativamente relacionado com a avaliação que elas fazem de suas próprias vidas é a idade de cada um, que influencia negativamente a satisfação com seus relacionamentos sociais. Dessa forma, essa menor satisfação com os relacionamentos manifesta-se naqueles adultos de idade avançada (acima de 27 anos), independentemente da renda familiar. Assim, quanto mais jovem a pessoa com deficiência, mais satisfeita mostra-se com o fator relações sociais. Talvez esse dado possa estar associado a uma maior abertura social que ocorre nos dias de hoje em relação à deficiência. A busca por estimulação desde a constatação da deficiência, a inclusão escolar ou o favorecimento do acesso de pessoas com deficiência ao mercado de trabalho são fatos que recentemente chamam a atenção da sociedade e a tornam mais permeável em relação à deficiência.

\section{Conclusões}

Com esta pesquisa, compreendeu-se que aquelas pessoas que possuem deficiência mental leve são capazes de falar da própria vida, de expressar toda a riqueza dos sentimentos que possuem, apresentando uma visão muito positiva da vida.

Elas percebem suas vidas como tendo qualidade, estão bastante satisfeitas e apresentam uma visão positiva sobre a vida, demonstrando que conhecem e entendem a realidade em que vivem, contradizendo crenças populares e até mesmo científicas de que não são capazes de fazê-lo.

Em nenhum estudo encontrado até o momento foi utilizado o WHOQOL-Bref com a população de pessoas com deficiência mental, demonstrando o caráter inovador desta pesquisa, bem como sua relevância para a área. Como os objetivos foram alcançados, ou seja, a população estudada compreendeu o propósito do instrumento utilizado, sugere-se a sua utilização em novos estudos com pessoas com deficiência mental leve para se conhecer mais detalhadamente o que esse grupo de pessoas pensa realmente, a forma como agem e os seus sentimentos. Sejam feitos estudos que dêem voz a essas pessoas, com o desenvolvimento até de instrumentos que captem os mais variados aspectos de suas vidas e que sejam adequados às suas necessidades.

Diante disso, talvez o papel da psicologia seja ouvir mais essas pessoas, buscando entendê-las e auxiliando-as a tornarem-se protagonistas de suas próprias vidas.

\section{Referências}

Almeida, A. C. \& Loureiro, S. R. (2000). Qualidade de vida no pósTMO. Em Multidisciplinaridade e reforma: temas, práticas e políticas. [Resumo]. Anais, VIII Ciclo de Saúde Mental (pp. 65-66). Ribeirão Preto: São Francisco Gráfica e Editora.

Assumpção Jr., F. B. (1991). A família e o deficiente mental. São Paulo: Ed. Paulinas.

Berglund, A. L. \& Ericsson, K. (2003). Different meanings of quality of life: a comparison between what elderly persons and geriatric staff believe is of importance. International Journal of Nursing Practice, 9, 112-119.

Bittencourt, Z. Z. L. C., Alves Filho, G., Mazzali, M., Santos, N. R. (2004). Quality of life in renal transplant patients: impact of a functioning graft. Revista de Saúde Pública, 38(5), 732-734.

Bullinger, M., Cachovan, M., Creutzig, A., Diehm, C., Gruss, J., Heidrich, H., Kirchberger, I., Loeprecht, H. \& Rogatti, W. (1996). Development of an illness-spesific instrument for assessment of quality of life in patientes with arterial occlusive diseade (Peripheral Arterial Occlusive Disease 86 Questionnaire). Vasa, 25(1), 32-40.

Bullinger, M., Von Mackensen, S., Fischer, K., Khair, K., Petersen, C., Ravens-Sieberer, U., Rocino, A., Sagnier, P., Tusell, J. M., Van Den Berg, M. \& Vicariot, M. (2002). Pilot testing of the "HaemoQoL" quality of life questionnaire for haemophiliac children in six European countries. Haemophilia, 8(2, Suplemento), 47-54.

Carvalho, M. C., Lima, L. A., Gonçalves, S. R., Barbosa, D. R. \& Fagundes, P. (2002). Qualidade de vida: Limitações de aplicação da escala WHOQOL-bref em pacientes psiquiátricos de longa permanência. Revista Brasileira de Psiquiatria, 24(2, Suplemento), 120-141.

Cummins, R. A. (2002). The validity and utility of subjective quality of life: a reply to Hatton and Ager. Journal of Applied Research in Intellectual Disabilities, 15, 261-268.

Emerson, E., Robertson, J. \& Wood, J. (2004). Levels of psychological distress experienced by family carers of children and adolescents with intellectual disabilities in an urban conurbation. Journal of Applied Research in Intellectual Disabilities, 17, 77-84.

Figueiredo, M. A. C. (1994). Profissionais de saúde e atitudes frente à síndrome da imunodeficiência adquirida: um estudo diferencial com base no modelo afetivo-cognitivo de Fishbein e Ajzen (1975). Tese de Livre Docência, Universidade de São Paulo, Ribeirão Preto.

Fleck, M. P. A., Leal, O. F., Louzada, S., Xavier, M., Chachamovich, E., Vieira, G., Santos, L. \& Pinzon, V. (1999). Desenvolvimento da versão em português do instrumento de avaliação de qualidade de vida da OMS (WHOQOL-100). Revista Brasileira de Psiquiatria, 21(1), 19-28. 
Fleck, M. P. A., Louzada, S., Xavier, M., Chachamovich, E., Vieira, G., Santos, L. \& Pinzon, V. (1999). Aplicação da versão em português do instrumento de avaliação da qualidade de vida da Organização Mundial de Saúde (WHOQOL-100). Revista de Saúde Pública, 33(2), 198-205.

Fleck, M. P. A., Louzada, S., Xavier, M., Chachamovich, E., Vieira, G., Santos, L. \& Pinzon, V. (2000). Aplicação da versão em português do instrumento abreviado de avaliação da qualidade de vida "WHOQOL-bref". Revista de Saúde Pública, 34(2), 178-183.

Fleck, M. P. A., Lima, A. F. B. S., Louzada, S., Schestaski, G., Henriques, A., Borges, V. R., Camey, S. \& Grupo LIDO (2002). Associação entre sintomas depressivos e funcionamento social em cuidados primários à saúde. Revista de Saúde Pública, 36(4), 431-438.

Gregory, N., Robertson, J., Kessissoglou, S., Emerson, E. \& Hatton, C. (2001). Factors associated with expressed satisfaction among people with intellectual disability receiving residential supports. Journal of Intellectual Disability Research, 45(4), 279-291.

Hastings, R. P., Allen, R., McDermott, K. \& Still, D. (2002). Factors related to positive perceptions in mothers of children with intellectual disabilities. Journal of Applied Research in Intellectual Disabilities, 15, 269-275.

Hatton, C. \& Ager, A. (2002). Quality of life measurement and people with intellectual disabilities: a Reply to Cummins. Journal of Applied Research in Intellectual Disabilities, 14, 195-217.

Kraemer, B. R., McIntyre, L. L. \& Blancher, J. (2003). Quality of life for young adults with mental retardation during transition. Mental Retardation, 41(4), 250-262.

Kuhnle, U., Bullinger, M. \& Schwarz, H. P. (1996). The quality of life in adult female patients with congenital adrenal hyperplasia: a comprehensive study of the impact of genital malformations and chronic disease on female patients life. European Journal of Pediatric, 155(7), 620-631.

Mancuso, C. A., Peterson, M. G. E. \& Charlson, M. E. (2001). Comparing discriminative validity between a disease-specific and a general health scale in patients with moderate asthma. Journal of Clinical Epidemiology, 54, 263-274.

Michelone, A. P. C. \& Santos, V. L. C. G. (2004). Qualidade de vida de adultos com câncer colorretal com e sem ostomia. Revista Latino-Americana de Enfermagem, 12(6), 875-883.

Moreno, A. B. \& Lopes, C. S. (2002). Quality of life assessment in laryngectomized patients: a systematic review. Caderno de Saúde Pública, 18(1), 81-92.

Organização Mundial de Saúde (1995). Classificação internacional das deficiências, incapacidades e desvantagens (Handicaps). Um manual de classificação das conseqüências das doenças (1980). 2a ed. Lisboa: Secretariado Nacional de Reabilitação.

Pruchno, R. A. \& Meeks, S. (2004). Health-Related Stress, Affect, and Depressive Symptoms Experienced by Caregiving Mothers of Adults with a Developmental Disability. Psychology and Aging, 19(3), 394-401.

Regen, M., Ardore, M. \& Hoffmann, V. M. B. (1993). Mães e filhos especiais. Relato de experiência com grupos de mães de crianças com deficiência. Brasília: Coordenadoria Nacional para Integração da Pessoa Portadora de Deficiência (CORDE).

Sabaz, M., Cairns, D. R., Lawson, J. A., Bleasel, A. F. \& Bye, A. M. E. (2001). The Health-Related Quality of Life of children with refractory epilepsy: a comparison of those with and without intellectual disability. Epilepsy, 42(5), 621-628.

Santi, L. N. (2003). Cuidando da saúde bucal do filho: o significado para um grupo de mães. Dissertação de Mestrado, Universidade de São Paulo, Ribeirão Preto.

Saupe, R., Nietche, E. A., Cestari, M. E., Giordi, M. D. M. \& Krahl, M. (2004). Qualidade de vida dos acadêmicos de enfermagem. Revista Latino-Americana de Enfermagem, 12(4), 636-642.

Schalock, R. L. (2000). Three decades of quality of life. Focuson-Autism-and-Other-developmental-Disabilities, 15(2), 116-127.

Schalock, R. L. (2004). The concept of quality of life: what we know and do not know. Journal of Intellectual Disability Research, 48(3), 203-216.

Schalock, R. L., Bonham, G. S. \& Marchand, C. B. (2000). Consumer based quality of life assessment: a path model of perceived satisfaction. Evaluation and Program Planning, 23, 77-87.

Schwartz, C. \& Rabinovitz, S. (2003). Life satisfaction of people with intellectual disability living in community residences: perceptions of the residents, theis parents and staff members. Journal of Intellectual Disability Research, 47(2), 75-84.

Schwartzman, L. Martin, G. L., Yu, C. T. \& Whiteley, J. (2004). Choice, degree of preference, and happiness indices with persons with intellectual disabilities: A surprising finding. Education and Training in Developmental Disabilities, 39(3), 265-269.

Shu, B. C., Lung, F. W. \& Huang, C. (2002). Mental health of primary family caregivers with children intellectual disability who receive a home care program. Journal of Intellectual Disability Research, 46(3), 257-263.

Souza, L. B. (2001). Organização do trabalho, Qualidade de Vida e Subjetividade: Um Estudo sobre Representações e Crenças de Profissionais do Campus da USP de Ribeirão Preto. Tese de Doutorado, Universidade de São Paulo, Ribeirão Preto.

Stockler, M. R., Osoba, D., Corey, P., Goowin, P. J. \& Tannock, I. F. (1999). Convergent Discriminative, and Predictive Validity of the Prostate Cancer Specific Quality of Life Instrument (PROSQOLI). Assessment and Comparison with Analogous Scales from the EORTC QLQ-C30 and a Trial-Specific Module. Journal of Clinical Epidemiology, 52(7), 653-666.

The Whoqol Group (1998a). Development of the World Health Organization WHOQOL-BREF Quality of Life Assessment Psychological Medicine, 28, 551-558.

The Whoqol Group. (1998b). The World Health Organization Quality of Life Assessment (WHOQOL): Development and General Psychometric Properties. Social Science and Medicine, 46(12), 1569-1585.

Velanovich, V. (2001). The quality of quality of life studies in general surgical journals. Journal of the American College of Surgeons, 193, 288-296.

Vickery, C. W., Blazeby, L. M., Conroy, T., Arraras, J., Sezer, O., Koller, M., Rosemey, D., Johnson, C. D. \& Alderson, D. (2001). Development of EORTC disease-specific quality of life module for use in patients with gastric cancer. European Journal of Cancer, 37, 966-971.

Walden, S., Pistrang, N. \& Joyce, T. (2000). Parents of adults with intellectual disabilities: Quality of life and experiences of caring. Journal of Applied Research in Intellectual Disabilities, 13, 62-76. 
Walsh, P. N. (2002). $3^{\text {rd }}$ annual conference of EASPD: 'Looking to a positive future - the best quality of life for ageing people with intellectual disabilities'. Journal of Intellectual Disability Research, 46(4), 361-363

Walsh, P. N., Heller, T., Shurf, N. \& Valk, H. S. L. (2001). Healthy ageing - adults with intellectual disabilities: women's health and related issues. Journal of Applied Research in Intellectual Disabilities, 14, 195-217.
World Health Organization. (2001). International classification of functioning, disability and health, Geneva: WHO.

Recebido em 27.10.2006 Primeira decisão editorial em 13.03.2007 Versão final em 11.09.2007 Aceito em 21.04.2008 